\title{
A Novel Method for Instantaneous, Quantitative Measurement of Molecular Mixing in Gaseous Flows
}

\author{
Hui Hu and Manoochehr M. Koochesfahani \\ Turbulent Mixing and Unsteady Aerodynamics Laboratory, Department of Mechanical Engineering \\ Michigan State University, East Lansing, 48824, Michigan. U.S.A. \\ Fax:1-517-353-7179_E-mail: huhui@egr.msu.edu
}

\begin{abstract}
We describe a novel method for making instantaneous, quantitative, planar measurements of fluid mixed at the molecular level in gaseous flows. The method relies on the effective oxygen quenching of the phosphorescence of luminescent tracers, such as acetone and biacetyl. The tracer's fluorescence emission is used to obtain information about the passive scalar, regardless of its molecular mixing state, whereas the phosphorescence emission from the same tracer displays mixing-state-dependant behavior and reveals the presence of moleculary-unmixed fluid. By combining the information from both fluorescence and phosphorescence signals, the instantaneous, quantitative measurements of molecularly-mixed fluid fraction can be obtained at each pixel of the detector. This method accomplishes the same objectives as the dual-tracer LIF method of King et al. (1997, 1999), but with a single tracer and a much reduced burden on the instrumentation and experimental setup. The new technique is demonstrated in a study of mixing in a forced acetone-seeded nitrogen jet discharging into ambient air. The instantaneous spatial maps of molecularly-mixed jet fluid fraction and jet fluid mixing efficiency are presented. The capability of the present technique to identify stirring at sub-resolution scale is also demonstrated.
\end{abstract}

\section{Introduction}

The mixing of two streams carrying different species is of great practical interest to numerous applications including chemical processing, material processing and aerospace propulsion. For example, in non-premixed combustion the total rate of heat release is governed by the mixing rate of fuel and air in a combustion chamber. Since the mixing of reactants at the molecular level is a prerequisite to chemical reaction, experimental methods that are capable of quantifying the instantaneous extent of molecular mixing are highly desirable. This is particularly important since the range of spatial and temporal scales involved in turbulent combustion do not currently allow a full computational model of the problem without the use of adhoc models.

A common approach for mixing studies is the use of Laser Induced Fluorescence (LIF) diagnostics for the quantitative mapping of a passive scalar field. In LIF a fluorescent tracer is 
premixed into one of the two mixing streams and the concentration of the tracer, after excitation by a laser, is measured using a CCD detector. In liquid-phase studies fluorescent dyes are often utilized (Dimotakis et al. 1983; Koochesfahani and Dimotakis, 1985), whereas in gas-phase investigations the use of fluorescent tracers such as biacetyl and acetone are now commonplace (Cruyningen et al. 1990; Lozano et al. 1992). LIF measures the average concentration of the fluorescent tracer contained within a small sampling volume determined by the spatial and temporal resolution characteristics of the measuring apparatus (i.e. detector pixel size, image ratio, etc.). The difficulty arises if the sampling volume is larger than the smallest mixing scales in the flow, as is usually the case in high Reynolds number flows. Under these circumstances, it is impossible to determine the true extent of molecular mixing within the measurement resolution volume and, as a result, the passive scalar technique tends to overpredict the actual amount of molecularly mixed fluid (Breidenthal, 1981; Koochesfahani and Dimotakis, 1986). High-resolution passive scalar LIF studies have been carried out while resolving the smallest mixing scales (Dahm et al., 1991; Su and Clemens, 1997). These are done by imaging a small region in the flow at the expense of sacrificing the overall view of the flow field and its largescale structures. We can estimate the smallest mixing scale by the Batchelor scale $\lambda_{B}=\delta \mathrm{Re}_{\delta}^{-3 / 4} S c^{-1 / 2}$, where $\delta$ represents the largest scale of the flow, $\mathrm{Re}_{\delta}$ the large scale Reynolds number, and $S c$ the Schmidt number. We note that, even for gas-phase flows where $S c$ is of order unity, the smallest mixing scale can be several orders of magnitude smaller than the large scale in high Reynolds numbers flows. The ability to optically image both scales simultaneously is limited by the pixel density of the detector array; if the imaging system is arranged to resolve the small scales, it would not be able to capture the largest feature of the flow at the same time. Being able to map the large scale passive scalar field is important to understanding and manipulating mixing at the molecular level since the overall evolution of the flow and entrainment of species into the mixing zone are often controlled by large scale dynamics.

The difficulties associated with the finite sampling volume can be solved by using diagnostics that rely on chemically-reacting techniques. In this case, the average amount of chemical product measured over the finite sampling volume gives the true indication of the extent of molecular mixing within that sampling volume. In order to isolate the potential effects of heat release and finite chemistry, many basic studies of mixing that utilize chemically-reacting methods use fast chemical reactions in the limit of low heat release (e.g. see Breidenthal, 1981 and Koochesfahani and Dimotakis, 1986 for liquid-phase applications and Mungal and Dimotakis, 1984 for gaseous mixing). In most chemically-reacting approaches only the product of the reaction is measured and not the distribution of the passive scalar reactants. Using fast chemistry and the probability density function (pdf) description of the scalar field, "flip experiment" methods have been devised to extract certain statistical properties of the scalar field from chemical product information (e.g. Koochesfahani et al., 1985; Koochesfahani and Dimotakis, 1985).

To study molecular mixing in gaseous flows Paul and Clemens (1993) and Clemens and Paul (1995) introduced a new method they called "cold chemistry." This method relies on the significant quenching of NO LIF signal by oxygen to provide a resolution-free measurement of the level of molecular unmixedness. The NO tracer is premixed into one of the two mixing streams containing no oxygen, whereas the other stream is ambient air. Since mixing of NO with even trace amounts of oxygen causes a large reduction of NO fluorescence intensity, the 
measured fluorescence intensity gives the amount of pure unmixed NO within each pixel of the detector. The instantaneous distribution of the amount of molecularly mixed fluid is not available in this method since a zero LIF signal could imply two streams that are completely mixed or simply pure unmixed fluid from the unseeded stream. Time-averaged properties of the molecular mixing field can be obtained, however, using the "flip experiment" approach and the pdf description of the scalar mixing field (Clemens and Paul, 1995; Island et al., 1996).

In another approach to directly image molecular mixing, Yip et al. (1994) described the method of sensitized phosphorescence. In this method the excited state molecules of one species (the donor) transfer energy through collisional interactions to another species (the acceptor), which then phosphoresces. Donor-acceptor pairs such as acetone-biacetyl or toluene-biacetyl have been considered. The quantitative utilization of this method is somewhat complex and requires detailed attention to the molecular interactions and energy transfer between the donor and acceptor molecules.

Recently, King et al. (1997, 1999) introduced a dual-tracer planar technique to provide instantaneous planar maps of molecular mixing in gaseous flows. The technique, which is a cold chemistry approach, relies on the simultaneous imaging of the LIF signals of acetone and NO tracers. As before, NO seeded into a nitrogen jet mixes with the oxygen in an air coflow and the resulting LIF signal quenching provides information on the pure unmixed jet fluid. Simultanesously, acetone LIF is used to obtain the concentration of the coflow fluid in the sampling volume, regardless of its molecular mixing state. By combining the information from these two LIF signals the instantaneous, quantitative measurements of molecularly mixed jetfluid can be obtained. This method requires the use of two tracers, two laser sources for excitation (one at $226 \mathrm{~nm}$ for NO and the other at $266 \mathrm{~nm}$ for acetone), generation of two coplanar laser sheets, and two spatially-aligned detectors with appropriate optical filters to minimize the cross contamination of the two LIF signals. Even though the experimental setup is relatively involved, the technique is quite powerful and has been effectively used in several studies.

In the present paper, we describe a novel method for making instantaneous, quantitative, planar measurements of fluid mixed at the molecular level. The method relies on the effective phosphorescence quenching by oxygen of luminescent tracers such as acetone and biacetyl. All previous methods based on fluorescence quenching rely on information obtained from the "intensity axis" of the emission process. In our approach, we rely also on the information contained in the "time axis" of the emission process, as oxygen quenching leads to several orders of magnitude reduction in the phosphorescence lifetime. This method accomplishes the same objectives as the dual-tracer LIF method of King et al., but with a single tracer and a much reduced burden on the instrumentation and experimental setup. The unmixedness information, which is derived from NO quenching in the dual-tracer method, is obtained here from the phosphorescence quenching of the same tracer that is used for scalar concentration measurements. In the sections that follow, the details of the new technique are given along with a demonstration of its application to mixing quantification in a forced round jet. 


\section{Description of the Experimental Technique}

The work here relies on the luminescence of popular tracers biacetyl and acetone, with a primary emphasis on acetone. The photophysics of these molecules have been described in Cruyningen et al. (1990) and Lozano et al. (1992) and the references therein. We need to distinguish between two types of luminescence processes, fluorescence and phosphorescence. The details and general properties of these processes can be found in texts on photochemistry (e.g. Turro, 1978; Ferraudi, 1988).

Fluorescence refers to the radiative process when a molecule transitions from a singlet excited state to its singlet ground state. Since singlet-singlet transitions are quantum mechanically allowed, they occur with a high probability, making fluorscence short-lived with short emission lifetimes on the order of 1-100 nanoseconds. The fluorescence lifetime of acetone, for example, is about 4 ns (Lozano et al., 1992). Phosphorescence, on the other hand, is a radiative process when a molecule transitions from a triplet excited state to its singlet ground state. Because such transitions are quantum mechanically forbidden, phosphorescence is long-lived with emission lifetimes that may approach milliseconds. The lifetime $\tau$ and quantum efficiency $\Phi$ (number of photons emitted per photons absorbed) of emission can be written as

$$
\begin{aligned}
& \tau=\frac{1}{k_{r}+k_{n r}+k_{q}[Q]}, \\
& \Phi=\frac{k_{r}}{k_{r}+k_{n r}+k_{q}[Q]} .
\end{aligned}
$$

In these expressions, the radiative $\left(k_{r}\right)$ and non-radiative $\left(k_{n r}\right)$ rate constants are intrintic properties of the excited state molecule, and the quenching rate constant $\left(k_{q}\right)$ and quencher concentration $[Q]$ account for the intermolecular reaction between the excited state molecule and a quencher molecule $Q$. As already mentioned, the quencher of interest to our work is oxygen $\mathrm{O}_{2}$. It follows from equations 1 and 2 that the lifetime and quantum efficiency in the presence of a quencher are connected to their corresponding values in the absence of the quencher (i.e. $\tau_{o}$ and $\Phi_{o}$ when $[Q]=0$ ) according to the Stern-Volmer relation

$$
\frac{\Phi_{o}}{\Phi}=\frac{\tau_{o}}{\tau}=1+\tau_{o} k_{q}[Q] .
$$

We note that the presence of a quencher leads to a reduction of the luminescence intensity and the emission lifetime. The fluorescence emission, due to its short lifetime, is usually little affected by the presence of a quencher. A good example is acetone whose fluorescence is unaffected by oxygen, making it a useful passive scalar tracer in gaseous flows. The long lifetimes of phosphorescence, on the other hand, makes it especially susceptible to quenching at extremely small quencher concentrations.

The effectiveness of oxygen quenching of acetone phosphorescence is illustrated in Figure 1. Acetone is seeded either into a nitrogen stream (from a commercial compressed $\mathrm{N}_{2}$ bottle, 
99.98\% purity) or ambient air stream. Excitation is provided by the 20 -ns pulse of a $\mathrm{XeCl}$ excimer laser (wavelength $\lambda=308 \mathrm{~nm}$ ) and the phosphorescence emission is measured as a function of time delay after the laser pulse. The minimum time delay is well beyond the 4-ns fluorescence lifetime of acetone, so that only the phosphorescence emission is captured by the detector. The measured emission intensity is an exponentially decaying function of the form

$$
I_{e m}=I_{o} e^{-t / \tau},
$$

where the lifetime $\tau$ refers to the time when the intensity drops to $37 \%$ (i.e. $1 / e$ ) of the initial intensity. The measured acetone phosphorescence intensities in air and nitrogen are shown in Figure 1 in log-linear form. The data in this figure indicate a reduction of phosphorescence lifetime from about $13 \mu \mathrm{s}$ in nitrogen to about $10 \mathrm{~ns}$ in air. This large reduction, by more than three orders of magnitude, is the basis of the present technique for establishing the state of mixing (acetone with $\mathrm{O}_{2}$ ) at the molecular level. For example, consider acetone that is molecularly mixed with air. The fluorescence image of acetone would give the concentration of acetone regardless of its mixing state, whereas a phosphorescence image acquired only $100 \mathrm{~ns}$ after laser excitation would have an intensity reduced by a factor of $e^{-10}$, providing an easy means of determining the state of molecular mixing.

Phosphorescence quenching is a function of the quencher concentration $\left[\mathrm{O}_{2}\right]$, which itself varies in the flow as the two streams mix. We will now establish the actual variation of phosphorescence emission with air mixture fraction. Consider the general case of the mixing between a nitrogen stream premixed with acetone tracer and ambient air stream (e.g. actonebearing nitrogen jet discharging into ambient air). In the absence of significant differential diffusion between acetone and nitrogen, acetone concentration $C$ is directly proportional to the nitrogen stream mixture fraction $f(f=0$ and 1 represent pure, or unmixed, air stream and nitrogen stream composition). The air mixture fraction $f_{\text {air }}$, given by $f_{\text {air }}=1-f$, provides information about the local oxygen concentration $\left[\mathrm{O}_{2}\right]$ in the mixture, using the fact that oxygen molar concentration in ambient air is about 0.2, i.e. $f_{\text {air }}=5\left[\mathrm{O}_{2}\right]$. The total phosphorescence intensity at a given point $I_{p}$ is given by

$$
I_{p}=I_{i} C \varepsilon \Phi_{p},
$$

where $I_{i}$ is the local incident laser intensity, $C$ the acetone concentration, $\varepsilon$ the absorption coefficient, and $\Phi_{p}$ the phosphorescence quantum efficiency (equations 2 and 3). The total phosphorescence intensity can be separately determined from the integration of equation (4) over all time, resulting in

$$
I_{p}=\tau I_{o} .
$$

Now consider capturing the acetone phosphorescence emission (see equation 4) by a gated intensified CCD detector where the integration starts at a delay time $t_{o}$ after the laser excitation pulse with a gate period $\Delta t$. The phosphorescence signal $S_{p}$ generated by the detector is then given by 


$$
S_{p}=\int_{t_{o}}^{t_{o}+\Delta t} I_{o} e^{-t / \tau} d t
$$

Using the equations given above, it can be shown that

$$
S_{p}=I_{i} f \varepsilon \Phi_{p}\left(1-e^{-\Delta t / \tau}\right) e^{-t_{o} / \tau}
$$

Using as the reference the corresponding phosphorescence signal $\left(S_{p}\right)_{o}$ in the pure unmixed nitrogen stream (where $f=1$, phosphorescence quantum efficiency $\left(\Phi_{p}\right)_{o}$ and lifetime $\tau_{o}$ ), we arrive at the following final expression for the normalized phosphorescence signal

$$
\frac{S_{p}}{\left(S_{p}\right)_{o}}=f \frac{\tau}{\tau_{o}} \frac{1-e^{-\Delta t / \tau}}{1-e^{-\Delta t / \tau_{o}}} e^{\left(t_{o} / \tau_{o}-t_{o} / \tau\right)} .
$$

The measured limiting acetone phosphorescence lifetimes in nitrogen and in air, shown in Figure 1 , can be used to determine the oxygen quenching rate $k_{q}$ according to equation (3), which is then used again to calculate the phosphorescence lifetime $\tau$ at any arbitrary air mixture fraction. The behavior of the normalized phosphorescence signal (equation 9) is plotted in Figure 2 versus the air mixture fraction for different detection delay times $t_{o}$ and gate periods $\Delta t$. Using the case of $\left(t_{o}=1 \mu \mathrm{s}, \Delta t=5 \mu \mathrm{s}\right)$, conditions for which we will present experimental results, we note that the phosphorescence signal provides a very sensitive means of detecting pure unmixed nitrogen/acetone composition. If the local air mixture fraction is higher than 0.05 , or nitrogen/acetone mixture fraction is lower than 0.95 , the signal drops by more than $3 \times 10^{-4}$, thereby providing a binary on/off indication of presence of unmixed/mixed fluid. Note that the sensitivity of the phosphorescence signal for detection of unmixed fluid is controlled by the choice of the two detection parameters time delay and gate period (see equation 9 and figure 2). The actual threshold for a binary on/off indication of unmixed/mixed fluid is dictated by the signal-to-noise ratio of the detector. For example, a CCD detector with 8 (useful) bits would produce a zero signal (a dark image) for air mixture fractions higher than 0.03 (nitrogen/acetone mixture fractions lower than 0.97 ) for the time delay $t_{o}=1 \mu$ s condition discussed.

The phosphorescence signal, equation (9), provides information on the local unmixedness of the nitrogen/acetone stream mixture fraction. The overall value of the mixture fraction, regardless of its molecular mixing state, is found from the fluorescence signal. Similar to equation (5), the total emitted fluorescence intensity at a given point can be written as

$$
I_{f}=I_{i} C \varepsilon \Phi_{f}
$$

where $I_{i}$ is the local incident laser intensity, $C$ the local acetone concentration, $\varepsilon$ the absorption coefficient, and $\Phi_{f}$ the fluorescence quantum efficiency. As before, the acetone concentration $C$ is directly proportional to the nitrogen stream mixture fraction $f$ in the absence of significant differential diffusion between acetone and nitrogen. Furthermore, as described earlier, the fluorescence quantum efficiency is a constant in this mixing problem and is not affected by the 
presence of oxygen. The total fluorescence signal $S_{f}$, which is captured by the detector integrating the fluorescence emission from the time of laser pulse over a gate period several fluorescence lifetimes long (gate period of $20 \mathrm{~ns}$ is sufficient, considering the 4-ns fluorescence lifetime of acetone), reduces to

$$
S_{f}=I_{i} f \varepsilon \Phi_{f}
$$

Using as the reference the corresponding fluorescence signal $\left(S_{f}\right)_{o}$ in the pure unmixed nitrogen stream (where $f=1$ ), we arrive at the following expression for the value of nitrogen/acetone mixture fraction in terms of the normalized fluorescence signal

$$
f=\frac{S_{f}}{\left(S_{f}\right)_{o}} .
$$

Equations (9) and (12) are the basis of the method described in this paper for the measurement of fluid mixed at the molecular level. We note that the potential contamination of the fluorescence signal by the phosphorescence emission of acetone can be made negligible by the appropriate choice of the integration period for capturing the fluorescence emission. Based on the quantum yield data provided in Lozano et al. (1992), we estimate that for a fluorescence detection gate period of $100 \mathrm{~ns}$ the acetone phosphorscence contributes less than $0.5 \%$ to the overall emission signal.

An analysis similar to above has been carried out for the biacetyl tracer. Results, not shown here, resemble those in Figure 2, but biacetyl is comparatively less effective than acetone in determining the level of unmixedness. In the next section, we describe the implementation and application of the present mixing quantification methodology.

\section{Experimental Setup}

In order to demonstrate the technique described above, we consider the measurement of the instantaneous map of the molecular mixing field in an excited gaseous jet flow. Figure 3 shows a schematic of the experimental setup. A blow-down nitrogen jet, seeded with acetone vapor, is discharged into ambient air using a high pressure nitrogen reservoir (gas cylinder) and appropriate pressure regulators, valves and flow management modules. The discharge nozzle has a sharp edge with a diameter $\mathrm{D}=2.54 \mathrm{~cm}$ at the exit. In order to seed the jet flow with acetone, the nitrogen stream is bubbled through liquid acetone in a seeding chamber. A flow capacitor is used to avoid the strong splashing of acetone in the seeding chamber when the jet flow is started impusively by opening the solenoid valve. An industrial loudspeaker mounted coaxially at the base of flow facility is used to excite the jet flow. A signal generator with power amplifier is used to supply the signal for the acoustical excitation.

For the present study, the jet exit speed was about $U=4.0 \mathrm{~m} / \mathrm{s}$, resulting in a jet Reynolds number $\mathrm{Re}=\mathrm{DU} / \mathrm{v} \approx 6,800$. The excitation frequency was set to $\mathrm{f}=80 \mathrm{~Hz}$, corresponding to a Strouhal number $\mathrm{St}=\mathrm{fD} / \mathrm{U}=0.5$. The jet flow was illuminated by a laser sheet which was 
formed from the beam of a XeCl excimer laser (wavelength $\lambda=308 \mathrm{~nm}$, energy $150 \mathrm{~mJ} / \mathrm{pulse}$, $20 \mathrm{~ns}$ pulse width) using appropriate optics. The acetone luminescence was acquired by a 12-bit high-resolution $(1280 \times 1024$ pixel) gated intensified CCD camera (PCO DiCAM-Pro) with a fast decay phosphor (P46). The detector was operated in the dual-frame mode, where two fullframe images of luminescence are acquired in quick succession from the same laser pulse. For these experiments, the first frame integrates the acetone fluorescence emission for a period of $100 \mathrm{~ns}$ starting at the laser pulse, whereas the second frame integrates the acetone phosphorescence for a period of $5 \mu$ s starting at a $1 \mu$ s delay after the laser pulse. The expected behavior of phosphorescence emission for these parameters have been discussed before (see Figure 2). The small amount of "ghost" image intensity (about 2\%) that is caused by the finite decay time of the phosphor for a $1 \mu$ s image separation has been corrected for in the results described. This affect can be completely eliminated using two separate, aligned, detectors.

The portion of the flow imaged on the detector is a region $100 \mathrm{~mm} \times 100 \mathrm{~mm}$ in the first four diameters of the jet. The spatial resolution of the measurement in the plane of illumination is, therefore, about $100 \mu \mathrm{m}$ per pixel. Since there is a time delay between the acquisition of fluorescence and phosphorescence images, the potential artifacts due to the flow displacement need to be considered. For these measurements, the $1 \mu$ s time separation leads to a maximum displacement of 0.04 pixel, which we consider negligible. For flows with very high local speeds, the issue of pixel matching between the fluorescence and phosphorescence images can complicate the interpretation of the instantaneous distribution of molecular mixing. The ensemble-averaged statistics of the mixing parameters will still be unambiguous, however.

The image pairs acquired for each laser pulse are corrected for any background intensity level and laser sheet intensity non-uniformity. The normalized fluorescence and phosphorescence images are then computed using the information from the pure unmixed region of the jet at the nozzle exit. Using a similar nomenclature as in King et al. (1999), the normalized fluorescence signal (equation 12) gives the spatial distribution of the "total" jet fluid mixture fraction, $f_{j, t}$, regardless of its molecular mixing state. The measured normalized phosphorescence signal gives the spatial distribution of the molecularly unmixed, pure, jet fluid mixture fraction $f_{j, p}$. The distribution of the molecularly-mixed part of jet fluid fraction $f_{j, m}$ is then found from

$$
f_{j, m}=f_{j, t}-f_{j, p} .
$$

The expression above is calculated on the pixel-by-pixel basis. A mixing efficiency $\eta$ can be defined for each pixel according to

$$
\eta=\frac{f_{j, m}}{f_{j, t}} .
$$

A value of $\eta=0$ indicates that the sampling volume imaged on a pixel contains only pure fluids from the two streams. A value of $\eta=1$ means that the contents of the sampling volume are completely mixed at the molecular level with a composition that has an air mixture fraction higher than the on/off quenching threshold (a value of 0.03 for the present experiments, see 
Section 2). A value in the range $0<\eta<1$ implies the existence of pure unmixed fluid at scales that are smaller than the image resolution, i.e. sub-resolution stirring.

\section{Experimental results and discussion}

Figure 4 shows a pair of typical instantaneous raw images of acetone fluorescence and phosphorescence (time delay $t_{o}=1 \mu \mathrm{s}$ ) emission in the excited gas jet flow. It is important to emphasize that these two luminescence images are from the same single laser pulse excitation, but acquired with a time delay relative to each other. The fluorescence image (figure 4a) depicts the rolling-up and shedding of an organized array of large-scale Kelvin-Helmholtz vortices as is expected in this forced jet. The phosphorescence image (figure $4 \mathrm{~b}$ ) reveals the spatial distribution of pure unmixed jet fluid. As described earlier, the molecular mixing of acetonebearing jet stream with even a very small amount of ambient air causes the phosphorescence signal to drop to below practical detection limits, providing a binary on/off indication of the presence of unmixed/mixed fluid. Note how effectively the raw phosphorescence image labels the unmixed jet fluid, even without the need for formal image processing. Comparison of the two images in Figure 4 reveals an intersting feature. The cores of the rolled-up vortices might at first appear to contain pure unmixed jet fluid on the basis of the fluorescence image. These cores are "dark" in the phosphorescence image, indicating that they are, in fact, completely mixed at the molecular level with the ambient air, thereby quenching the phosphorescence emission.

The various quantitative measures of the mixing field are derived from the image pair just described using the procedures already discussed. These four measures include the total jet fluid fraction $f_{j, t}$, the pure unmixed jet fluid fraction $f_{j, p}$, the molecularly-mixed jet fluid fraction $f_{j, m}$, and mixing efficiency $\eta$. The instantaneous spatial distributions of these measures are illustrated in Figure 5. The images shown are very similar to those obtained by King et al. $(1997,1999)$ using the dual-tracer method. We note in Figure 5(c) that, as expected, molecular mixing between the jet fluid and ambient occurs within the vortex cores and braid regions in the mixing layer of the near-field jet.

Sample quantitative transverse profiles of the four mixing measures are extracted from the images in Figure 5 at the two downtream locations $\mathrm{X} / \mathrm{D}=1.5,3.5$ and the results are given in Figure 6 . The enlarged views are also shown to highlight the details within the regions at these two downstream locations marked by windows $\mathrm{A}$ and $\mathrm{B}$. The profiles at $\mathrm{X} / \mathrm{D}=1.5$ show that the molecularly-mixed jet fluid fraction, $f_{j, m}$, in the core regions of the Kelvin-Helmholtz vortices is relatively high $\left(f_{j, m} \approx 0.95\right)$. The mixing efficiency is almost unity in these regions, indicating that the mixture of jet and ambient fluids in the cores is completely mixed at the molecular level as early as 1.5 diameters away from the nozzle exit. At the further downstream location $\mathrm{X} / \mathrm{D}=$ 3.5 , more ambient air is engulfed in the mixing zone, and the molecularly-mixed jet fluid fraction in the core regions decreases. By this location, complete mixing at molecular level $(\eta=1)$ has been achieved between jet fluid and ambient air over most of the jet width. The enlarged views showing the details inside windows $A$ and $B$ (figures $6 c, d$ ) indicate areas on the jet side of the mixing layer where the mixing efficiency is $0<\eta<1.0$. In Figure 6(c), we note that in the range $0.22<\mathrm{Y} / \mathrm{D}<0.24$ the total jet fluid fraction equals one, within the noise limit of the measurement, and would suggest completely unmixed jet fluid. The molecularly-mixed jet fluid 
fraction is, however, greater than zero indicating that in reality some portion of the fluid is mixed at the molecular level. An interesting feature is depicted in Figure $6(d)$ in the region near $\mathrm{Y} / \mathrm{D}=$ -0.03 . Note that the total jet fluid fraction is noticeably lower than unity; this would imply complete mixing within the resolution constraints of the passive scalar measurement. However, the mixing efficiency is also less than unity, implying the existence of pure unmixed fluid at scales that are below the pixel resolution. This is an example of sub-resolution stirring, which causes the known overprediction of the true extent of molecular mixing with under-resolved passive scalar measurements.

\section{Conclusions}

A novel method has been described for conducting instantaneous, quantitative, planar measurements of molecular mixing in a gaseous flow. The technique is a "cold chemistry" approach and takes advantge of the effective quenching of the phosphorescence of tracers, such as acetone and biacetyl, by trace amounts of oxygen. The laser-induced fluorescence emission, which is not quenched by oxygen, provides information on the behavior of the passive scalar in a gaseous flow, regardless of its molecular mixing state. The laser-induced phosphorescence signal, which is greatly quenched by oxygen, displays mixing-state-dependant behavior and reveals the presence of moleculary-unmixed fluid. By combining information from both flourescence and phosphorescence signals, the instantaneous, quantitative measurements of molecularly-mixed fluid fraction can be obtained at each pixel of the detector. With this approach, the correct estimate of molecular mixing is obtained even if the smallest mixing scales are not resolved. The method described here uses a single tracer, single laser, and a single (dualframe) detector. It accomplishes the same objectives as the dual-tracer LIF method of King et al. (1997, 1999), but with a much reduced burden on the instrumentation and experimental setup.

The implementation and application of the new technique are demonstrated in a study of mixing in a forced acetone-seeded nitrogen jet discharging into ambient air. The instantaneous maps of molecularly-mixed jet fluid fractions and sub-resolution stirring (in the form of mixing efficiency) have been presented in the near field of the forced jet flow. The results demonstrate the capability of this novel technique for obtaining instantaneous, quantitative measurements of molecular mixing, while at the same time visualizing large-scale mixing structures in the flow.

\section{Acknowledgments}

This work was supported by the MRSEC Program of the National Science Foundation, Award Number DMR-9809688. 


\section{References}

Breidental, R. (1981) "Structure in turbulent mixing layers and wakes using a chemical reaction," J. Fluid Mech,. Vol. 109, 1-14.

Clemens, N. T. and Paul, P. H. (1995) "Scalar measurements in compressible axisymmetric mixing layers," Phys. Fluids, Vol. 7, No.5, 1071-1081.

Cruyningan, I. van, Lozano, A., and Hanson, R. K. (1990) "Quantitative imaging of concentration by planar laserinduced fluorescence," Exp. Fluids, Vol. 10, No. 1, 41- 49.

Dahm, W. J. A., Southerland, K. B. and Buch, K. A. (1991) "Direct, high resolution, four-dimensional measurements of the fine scale structure of $\mathrm{Sc}>>1$ molecular mixing in turbulent flows," Phys. Fluids A, Vol. 3, No. 5, 1115-1127.

Dimotakis, P. E., Miake-Lye, R. C. and Papantoniou, D. A. (1983) "Structure and dynamics of round turbulent jets," Phys. Fluids, vol. 26, 3185-3192.

Ferraudi, G. J. (1988) Elements of Inorganic Photochemistry, Wiley-Interscience, New York.

Island T. C., Urban, W. D. and Mungal, M. G. (1996) "Quantitative scalar measurements in compressible mixing layers," AIAA paper No. AIAA-96-0685

King, G. F., Lucht, R. P. and Dutton, J. C. (1997) "Quantitative dual-tracer planar laser-induced flourescence measurements of molecular mixing," Optics Lett., Vol 22, No. 9, 633-635.

King, G. F., Dutton, J. C. and Lucht, R. P. (1999) "Instantaneous, quantitative measurements of molecular mixing in the axisymmetric jet near field," Phys. Fluids, Vol.11, No. 2, 403-416.

Koochesfahani, M. M., Dimotakis, P. E. and Broadwell, J. E. (1985) "A flip experiment in a chemically reacting turbulent mixing layer," AIAA J., Vol. 23, No. 8, 1191-1194.

Koochesfahani, M. M. and Dimotakis, P. E. (1985) "Laser induced fluorescence measurements of mixed fluid concentration in a liquid plane shear layer," AIAA J., Vol. 23, No. 11, 1700-1707.

Koochesfahani M. M. and Dimotakis P. E. (1986) "Mixing and chemical reactions in a turbulent liquid mixing layers," J. Fluid Mech., Vol. 170, 83-112.

Lozano, A., Yip, B., and Hanson, R. K. (1992) "Acetone: a tracer for concentration measurement in gaseous flows by planar laser-induced flourescence," Exp. Fluids, Vol. 13, 369-376.

Mungal, M. G. and Dimotakis, P. E. (1984) "Mixing and combustion with low heat release in a turbulent shear layer," J. Fluid Mech. Vol. 148, 349-382.

Paul, P. H. and Clemens, N. T. (1993) "Subresolution measurements of unmixed fluid using electronic quenching of NO A ${ }^{2} \Sigma^{+}, "$ Optics Lett., Vol. 18, No. 2, 161-163.

$\mathrm{Su}$, L. and Clemens, N. T. (1997) "Measurements of three-dimensional scalar dissipation rate in gas-phase turbulent jets," AIAA paper No. AIAA-97-0074.

Turro, N. J. (1978) Modern Molecular Photochemistry, Benjamin/Cummings, Menlo Park, CA.

Yip, B., Lozano, A. and Hanson, R. K. (1994) "Sensitied phosphorescence: a gas phase molecular mixing diagnostic," Exp. Fluids, Vol.17, 16-23. 


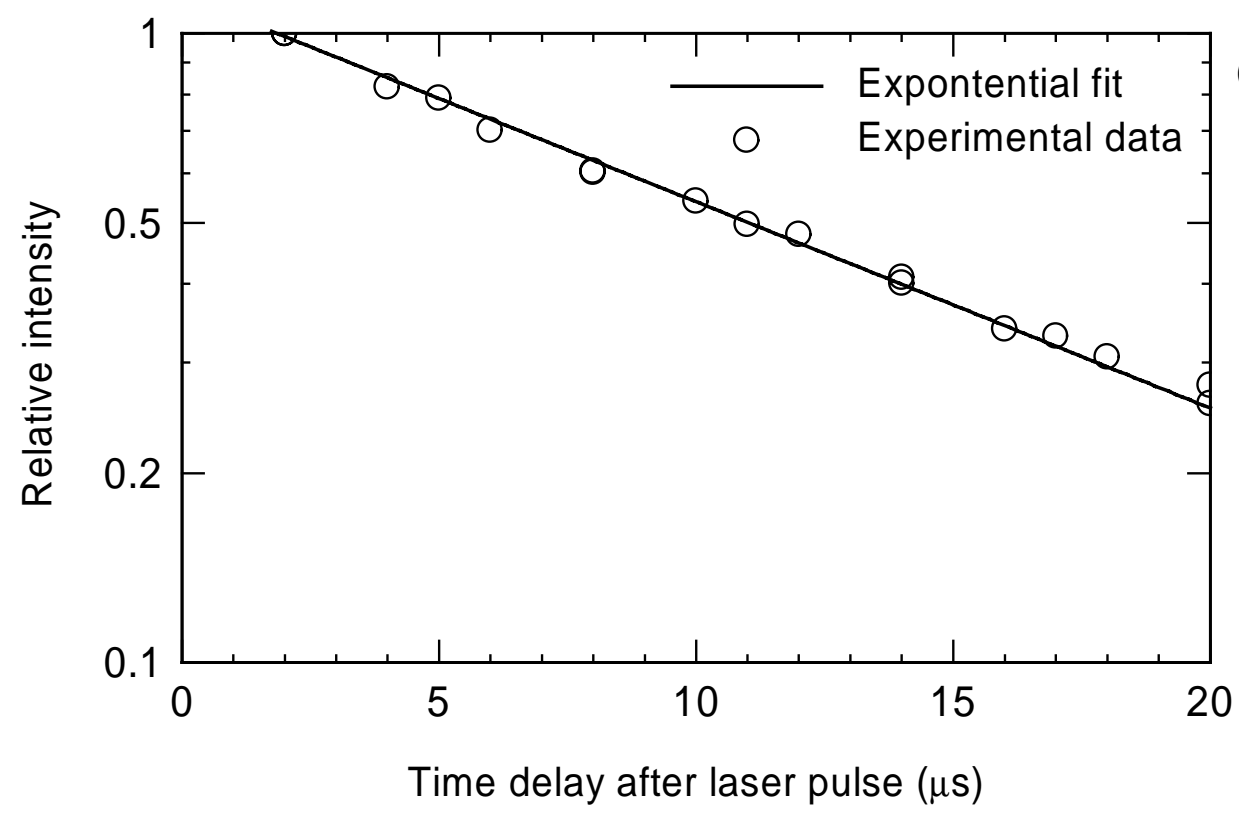

(a)

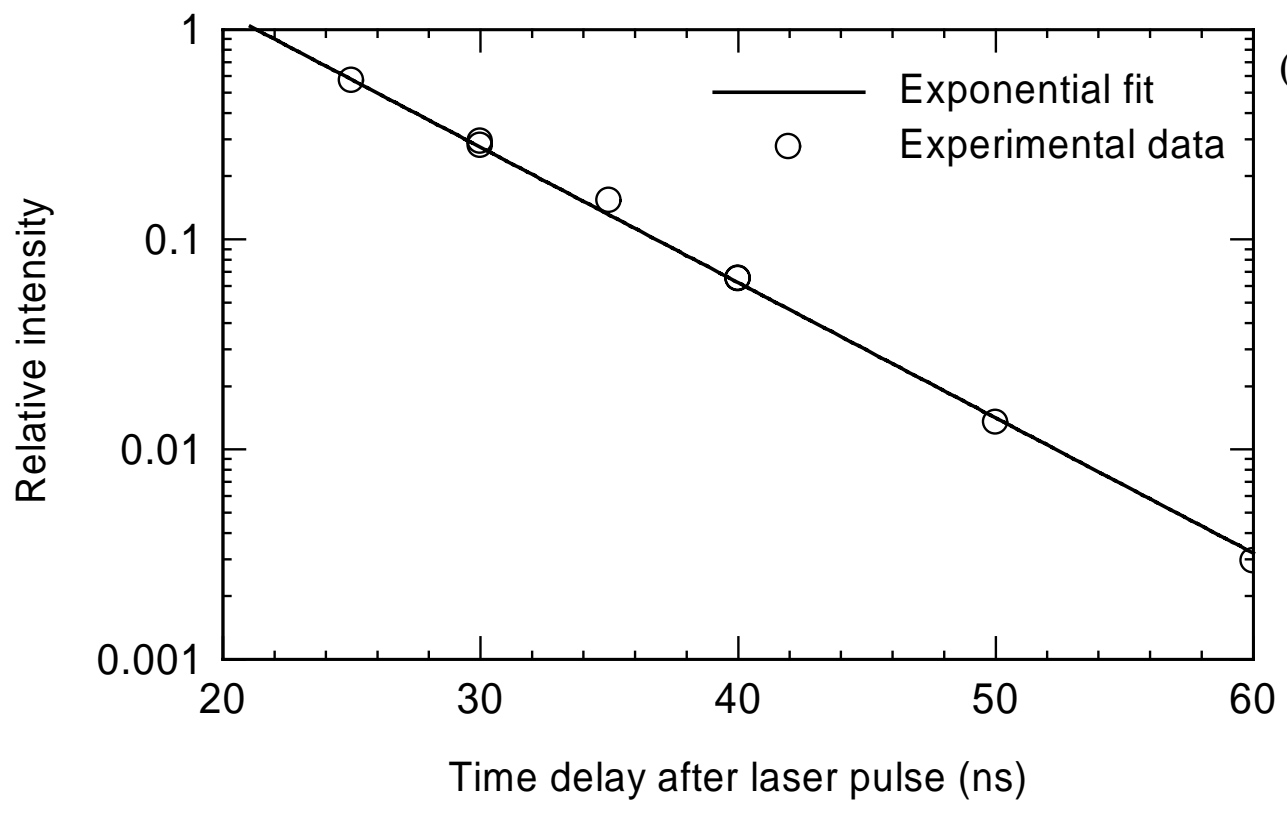

Figure 1. Decay of acetone phosphorescence emission with time; (a) acetone carried in nitrogen, (b) acetone carried in ambient air. 


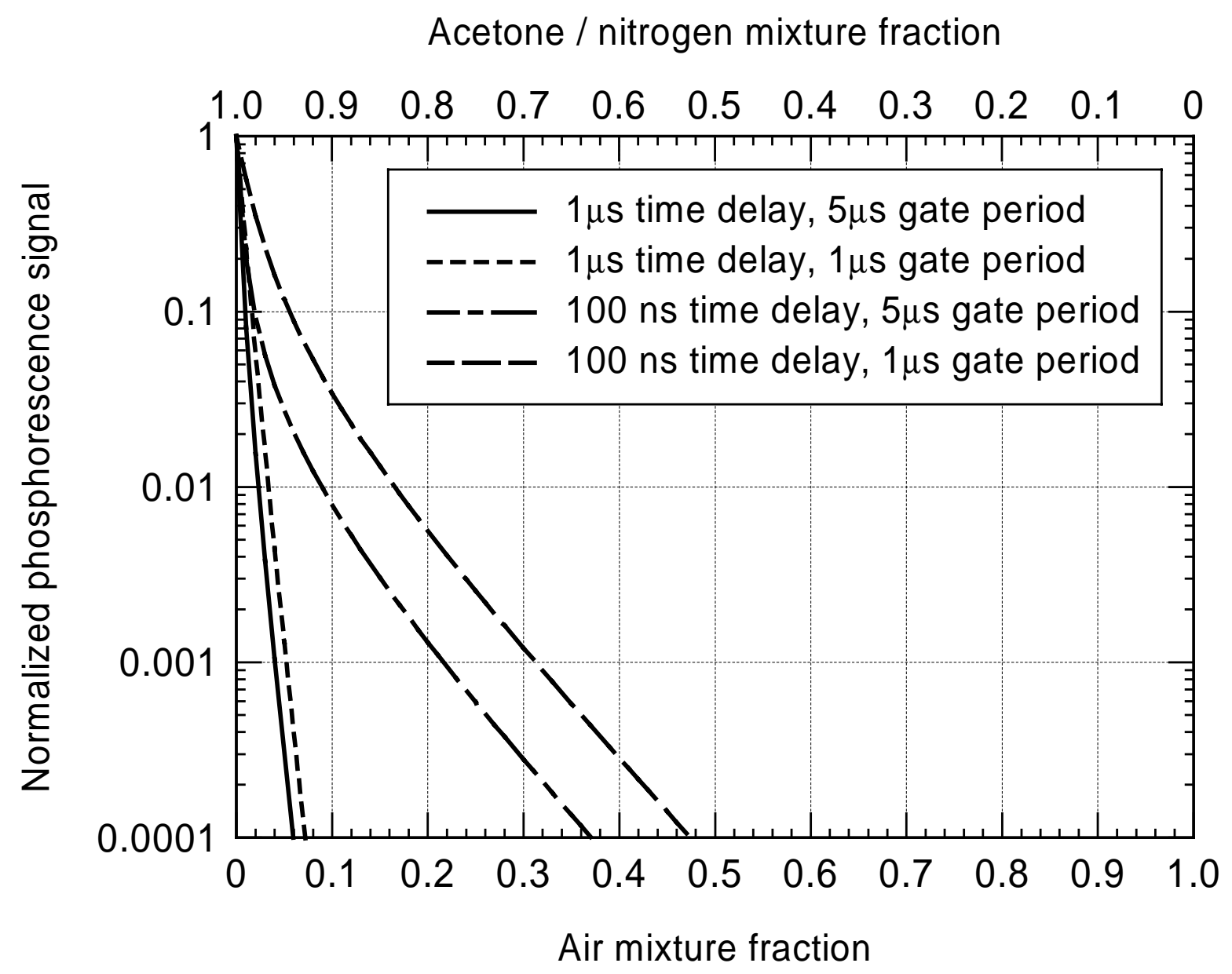

Figure 2. Normalized acetone phosphorescence signal versus air mixture fraction. 


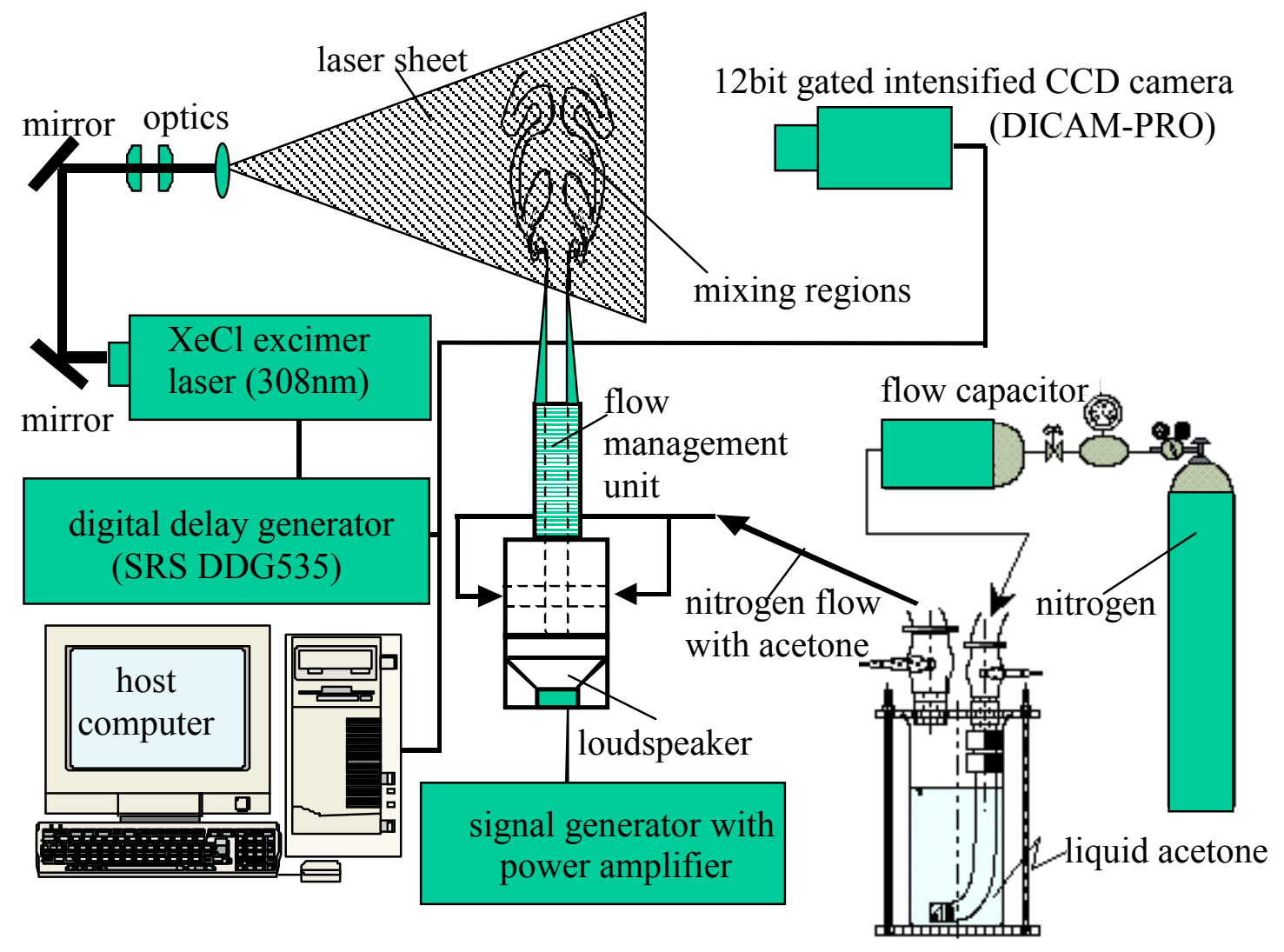

Figure 3. Schematic of experimental setup. 


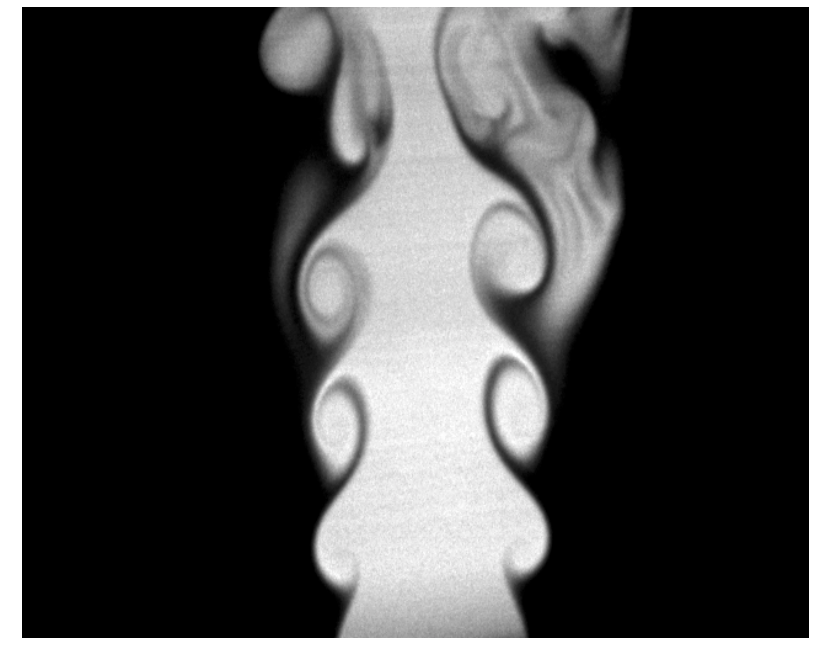

(a) fluorescence image

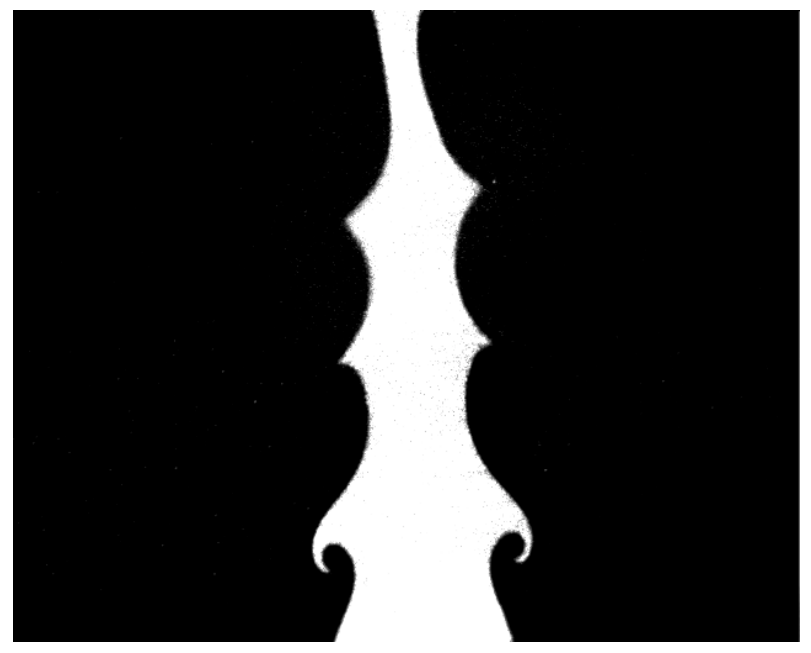

(b) phosphorescence image, time delay $t_{o}=1 \mu \mathrm{s}$

Figure 4. Typical fluorescence and phosphorescence raw (unprocessed) images obtained from one laser pulse. 


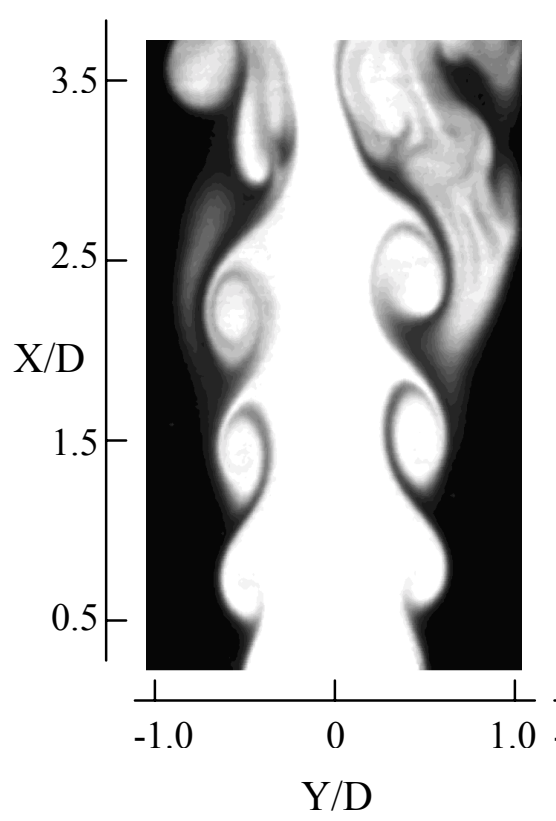

(b) Total jet fluid fraction $f_{j, t}$

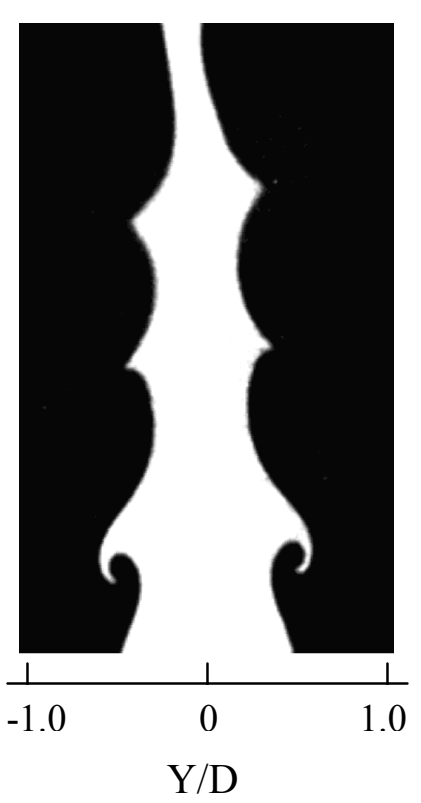

(a) Pure jet fluid fraction $f_{j, p}$
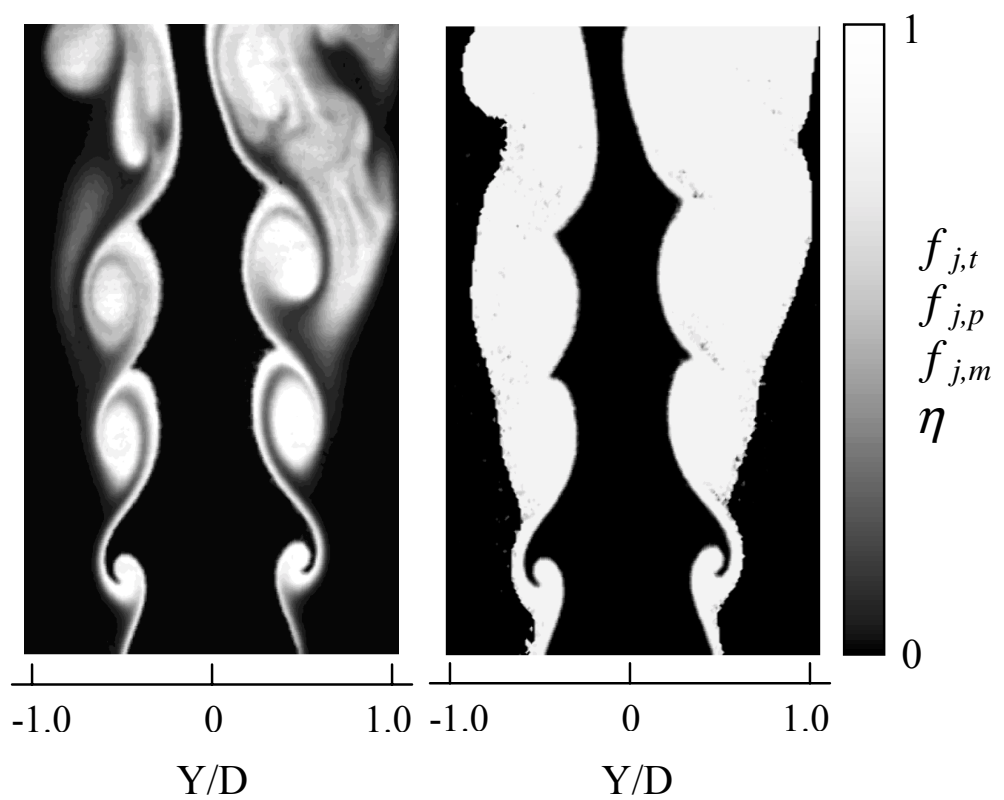

(c) Molecularly-mixed jet fluid fraction $f_{j, m}$ (d) Mixing efficiency $\eta$

Figure 5. The instantaneous spatial map of various mixing variables. 

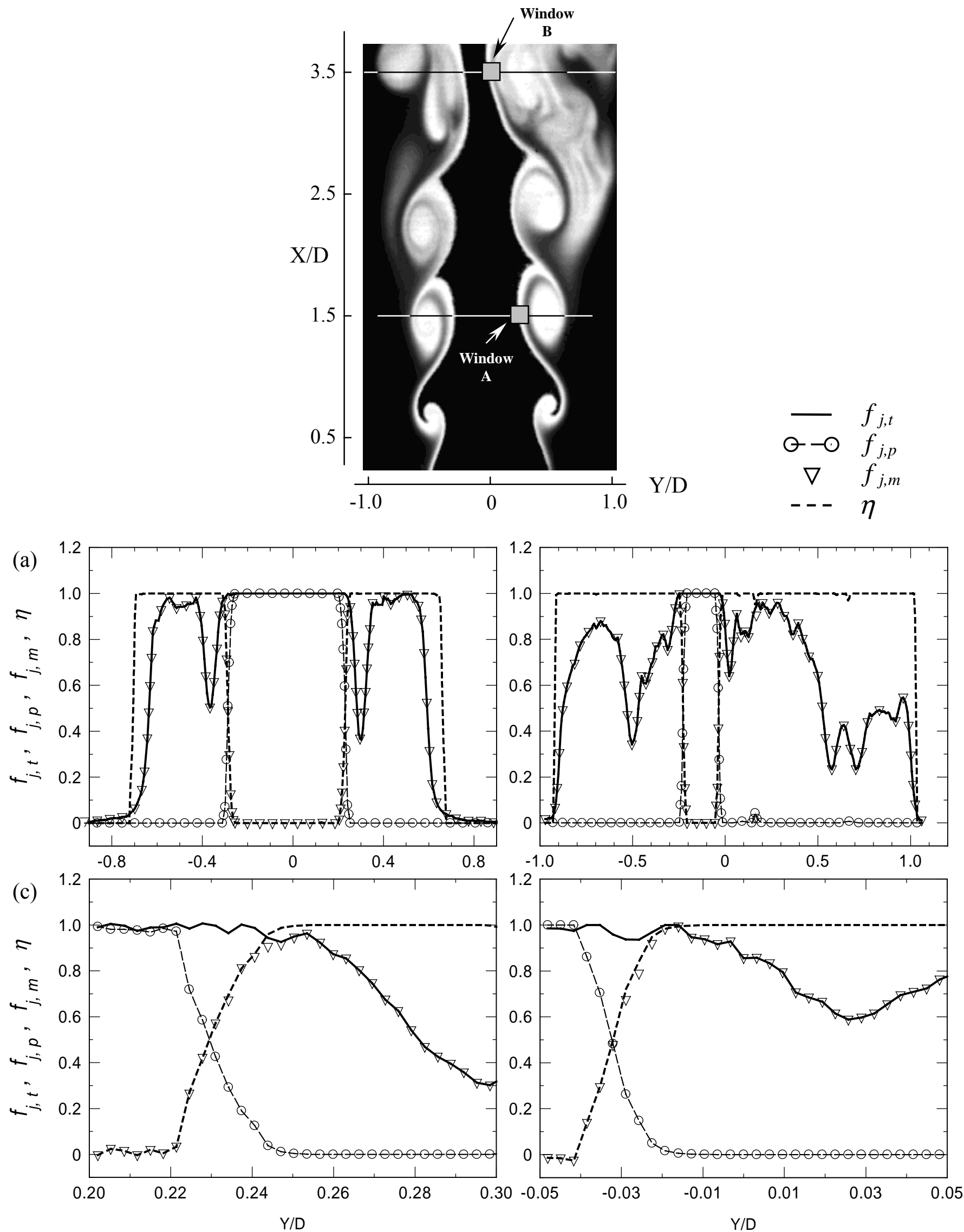

(b)

Figure 6. Quantitative transverse profiles of various mixing variables; (a) $\mathrm{X} / \mathrm{D}=1.5$, (b) $\mathrm{X} / \mathrm{D}=3.5$, (c) window $\mathrm{A}$, (d) window $\mathrm{B}$. 